\title{
On bias in magnitude scaling and some conjectures of Stevens
}

\author{
LAWRENCE T. DECARLO \\ Teachers College, Columbia University, New York, New York
}

\begin{abstract}
Bias in magnitude scaling can be viewed as involving deviations of judgments from proportionality. A model of bias is shown to provide a theoretical basis for Stevens's conjecture about geometrically averaging magnitude estimation and magnitude production exponents in order to obtain an estimate of the psychophysical exponent. An overlooked result is that one can also obtain an estimate of the magnitude of the bias. Examples from several well-known studies are presented. The bias is also shown to vary in response to experimental manipulation of the stimulus range. Aspects of predicting exponents across experiments are clarified, and a new prediction is examined. The model of bias fills some theoretical gaps in magnitude scaling and clarifies underlying assumptions and predictions.
\end{abstract}

In a magnitude estimation (ME) experiment, different intensities of a stimulus are presented to a participant, who attempts to provide numerical responses that are proportional to his or her sensation magnitudes (see Gescheider, 1997; Stevens, 1986). Stevens noted that data from $\mathrm{ME}$ are consistent with a simple power function,

$$
N=\alpha S^{\beta}
$$

in which $N$ is the observed numerical response, $S$ is the physical value of the presented stimulus intensity, $\alpha$ is a proportionality constant, and $\beta$ is the psychophysical exponent. Note that the purpose here is to illustrate certain theoretical relations, and so the presentation is simplified by not including error terms in the models (cf. DeCarlo, 1994, 2003), although their inclusion would not change any of the basic results presented below. Some statistical estimation issues also arise, but they are not of central interest here, although they are noted where appropriate.

It is important to recognize that two basic assumptions underlie Equation 1. One involves the relation between sensation magnitude and stimulus intensity - that is, the psychophysical function, which Stevens argued is a power function,

$$
\Psi=S^{\beta},
$$

in which $\Psi$ represents the sensation magnitude (note that Equation 2 does not include a proportionality constant). A second basic assumption involves the relation between sensation magnitude and observed responses - that is, the judgmental model. Stevens assumed a simple model of proportional judgment,

$$
N=\alpha \Psi
$$

Correspondence should be addressed to L. T. DeCarlo, Department of Human Development, Box 118, Teachers College, Columbia University, 525 West 120th Street, New York, NY 10027 (e-mail: decarlo@tc.edu). in which $\alpha$ is a proportionality constant. The meaning of the proportionality constant is clarified when one considers exactly how participants might go about making proportional judgments, as discussed below. Note that Equations 2 and 3 together give Equation 1.

Equations 2 and 3 can be viewed as theoretical models underlying Stevens's power law. Generalizations of these models have been considered in the psychophysical literature, particularly in research concerned with sequential effects, where various dynamic extensions have been considered. For example, the psychophysical function (Equation 2) has been generalized to allow for possible stimulus context effects (e.g., Cross, 1973; DeCarlo $\&$ Cross, 1990), whereas the judgmental model of Equation 3 has been generalized to allow for effects of changes in the proportionality constant over trials, due to the use of different reference points (e.g., DeCarlo, 1990, 1994, 2003; DeCarlo \& Cross, 1990). Although the model of bias presented below can easily be incorporated into dynamic models, dynamic generalizations are not the focus here (and they do not change the main results presented below). Rather, a simple generalization of the judgmental model is considered; the generalization allows for deviations from proportional judgment, the consequences of which are examined in detail.

It is widely recognized that the model of proportional judgment given by Equation 3, although ideally desired, is somewhat restrictive. For example, it has long been known that participants' responses are subject to various kinds of "biases" (e.g., Poulton, 1979), such as shortening or expanding the range of responses, effects of stimulus range, possible number preferences, and so on. Bias, from the present perspective, simply means that responses are not strictly proportional to sensation magnitudes. This, in turn, means that the judgmental model of Equation 3 should be generalized. The present note shows one way to generalize the judgmental model and examines its implications. It is shown that basic aspects of bias can be 
captured via a simple power function combined with a process model of proportional judgment. The resulting model provides a theoretical basis for Stevens's conjecture about geometrically averaging the exponents from ME and magnitude production (MP) experiments. In addition, the model of bias (1) shows how to obtain an estimate of the magnitude of the bias, which has been overlooked up to this point, (2) quantifies the regression effect, (3) clarifies assumptions underlying several of Stevens's conjectures, and (4) clarifies details of how response bias affects proportional judgments in ME and MP. The model of bias is also applied to cross-modality matching (CMM) experiments, and several additional important results are derived. For example, with regard to predicting exponents across experiments, on which Stevens placed great emphasis, the present derivations clarify exactly when bias will or will not be removed; some examples are considered below. The generalized judgmental models offered here fill some gaps in the theory of magnitude scaling, particularly with respect to clarifying the role of response bias in ME, MP, and CMM.

\section{PROPORTIONAL JUDGMENT}

\section{Magnitude Estimation}

As shown by Equation 3, a basic assumption in magnitude scaling experiments is that participants' judgments are proportional to their sensation magnitudes. A firmer theoretical basis for Equation 3 can be obtained, however, by considering how they might actually go about making proportional judgments. This, in turn, necessitates an understanding of the role of reference points in proportional judgment. For example, one approach is to assign a reference response, say $N_{0}$, to a reference sensation, say $\Psi_{0}$. The reference response is referred to as the modulus in psychophysics, whereas the physical value of the stimulus that corresponds to the reference sensation (denoted $S_{0}$ below) is referred to as the standard. On each trial, participants compare the sensation from the just-presented stimulus, say $\Psi$, with the reference sensation $\Psi_{0}$ and give a response $N$, so that the relation between $N$ and $N_{0}$ reflects the relation between $\Psi$ and $\Psi_{0}$. If the relations are proportional, the judgmental model can be written as

$$
N / N_{0}=\Psi / \Psi_{0} .
$$

Equation 4 simply shows one way to make proportional judgments in ME. For example, it follows from Equation 4 that if the current sensation $\Psi$ is twice the strength of the reference sensation $\Psi_{0}$, the current number $N$ should be twice as large as the reference number $N_{0}$. Rearranging Equation 4 gives

$$
N=\left(N_{0} / \Psi_{0}\right) \Psi=\alpha_{0} \Psi,
$$

which shows that the proportionality constant $\alpha_{0}$ depends on the reference response $N_{0}$ and the reference sensation $\Psi_{0}$ used by the participant. ${ }^{1}$ Substituting the psychophysical function (Equation 2) for $\Psi$ and $\Psi_{0}$ in the above gives

$$
N=\left(N_{0} / S_{0}^{\beta}\right) S^{\beta}=\alpha S^{\beta},
$$

in which $S_{0}$ is the stimulus intensity that corresponds to the reference sensation $\Psi_{0}$. Thus, Equations 2 and 4 provide a theoretical basis for Stevens's power law, Equation 1.

Equation 6 is important because it clarifies several aspects of Stevens's power law. For example, Equation 6 shows that the proportionality constant $\alpha$ depends on three factors: the reference response $\left(N_{0}\right)$ chosen by the participant, the reference sensation chosen by the participant, and the choice of units of measurement for the stimulus (which determines $S_{0}$ ). The effect of arbitrary units of measurement was noted by Rule (1993) as a reason why the intercept (i.e., $\log \alpha$ ) is not simply a subjective unit of measurement. This is clearly shown by Equation 6 , in that the proportionality constant is $\alpha=N_{0} / S_{0}^{\beta}$, which shows that $\alpha$ depends on the units of measurement for the physical stimulus (through $S_{0}$ ). Thus, the meaning of the proportionality constant is clarified by considering the process by which participants might go about making proportional judgments (i.e., Equation 4).

\section{Magnitude Production}

The task in MP is the opposite of that in ME. In MP, participants vary a physical stimulus in order to produce sensations that are proportional to presented numbers. Analogous to Equation 4, the judgmental model can be written as

$$
\Psi^{\prime} / \Psi_{0}^{\prime}=N / N_{0},
$$

in which $N$ is the number presented on the current trial, $N_{0}$ is a reference number used by the participant, and the primes on $\Psi$ and $\Psi_{0}$ indicate that the sensations are produced by the participant (i.e., by varying $S$; see DeCarlo, 2003). Equation 7 simply shows one way to make proportional judgments in MP. To start, one assigns a reference sensation, $\Psi_{0}^{\prime}$, to a reference number, $N_{0}$. For example, a participant might choose the number $10\left(N_{0}\right)$ and associate a certain produced loudness sensation $\left(\Psi_{0}^{\prime}\right)$ with it; the number and loudness then together serve as a reference point for comparative judgment, as in ME above. If the experimenter then presents the number 20 $(N)$, say, in order to make a proportional judgment, the participant has to produce a sensation $\Psi^{\prime}$ that is twice the intensity of the reference sensation $\Psi_{0}^{\prime}$. Thus, the judgmental model of Equation 7 is simply a clarification of the process leading to proportional judgment in MP.

\section{BIAS AS DEVIATIONS FROM PROPORTIONAL JUDGMENT}

As noted above, bias can be viewed as indicating that participants' judgments deviate from proportionality in some way. This, in turn, implies that the judgmental models given above as Equations 4 and 7 for ME and MP, 
respectively, should be generalized. Of course, there are many ways to do this; I comment at the end of the present article on a general approach. This section shows that a simple power function generalization of the judgmental models has several important and heretofore overlooked implications. Although power function generalizations of judgmental models have previously been considered (e.g., Cross, 1974; Curtis, Attneave, \& Harrington, 1968; Teghtsoonian, 1973), the generalizations have differed in various ways from the present approach.

\section{Bias in Magnitude Estimation}

One way to generalize Equation 4 to allow for bias is as follows:

$$
N / N_{0}=\left(\Psi / \Psi_{0}\right)^{b},
$$

in which $b$ is a bias parameter. If $b=1$, there is no bias and responses are proportional to sensation magnitudes. If $b \neq 1$, there is bias and responses deviate from proportionality. For example, if $b<1$, sensation ratios are judged as being less extreme, whereas if $b>1$, sensation ratios are judged as being more extreme. To illustrate, the top panel of Figure 1 shows the effects of no bias (i.e., $b=1)$ and bias less than unity $(b=0.6$; note that the range is restricted on the right so that the effect for ratios less than unity is more clearly seen); the lower panel shows the relations on logarithmic coordinates. The figure shows that, for $b<1$, sensation ratios greater than unity or less than unity are judged as being less extreme. Thus, the bias can be viewed as representing a constriction or shortening of the response range: The numbers are too small for $\Psi / \Psi_{0}>1$ and too large for $\Psi / \Psi_{0}<1$, so the participant tends to avoid extremes, which is in line with Stevens's "comfort" and "caution" explanations of the regression effect (1986, pp. 273-274; see also Stevens \& Greenbaum, 1966; Teghtsoonian \& Teghtsoonian, 1978). Note, however, that the exact description of the nature of the response bias depends on where the reference sensation $\Psi_{0}$ is located. For example, for $\Psi_{0}$ in the middle of the presented sensation range, bias less than unity means that the numbers are both too small and too large, as just described. However, if $\Psi_{0}$ is located at the low end of the presented sensation range (i.e., so that the sensation ratios are all greater than or equal to unity), bias less than unity means that the participant does not give large enough numbers, whereas if $\Psi_{0}$ is located at the high end of the sensation range (i.e., so that the sensation ratios are less than or equal to unity), small enough numbers are not given. In other words, whether the shortening is in both directions or only one direction depends on where $\Psi_{0}$ is located (which is not known). Thus, the simple generalization given by Equation 8 can handle different types of deviations from proportionality (including several types of biases described by Poulton, 1979).

Substituting the psychophysical function (Equation 2) into Equation 8 and rearranging gives

$$
N=\left(N_{0} / S_{0}^{b \beta}\right) S^{b \beta},
$$
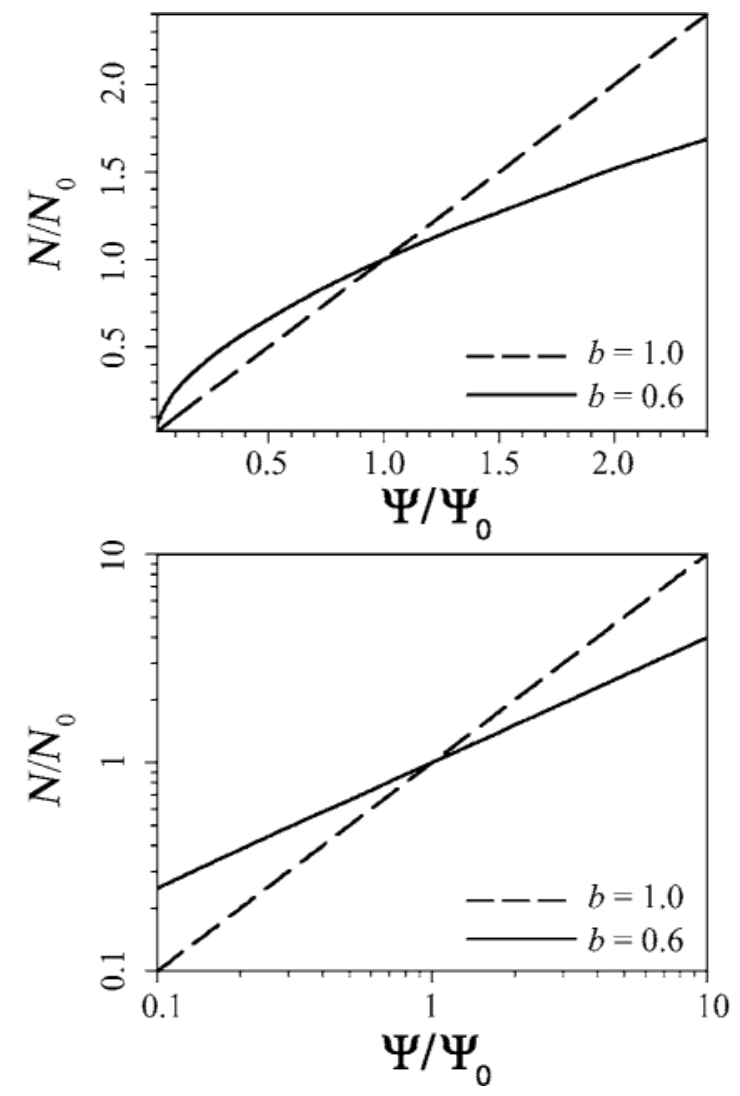

Figure 1. An illustration of the effects of response bias in magnitude estimation. The $x$ axis shows unobserved sensation ratios, and the $y$ axis shows observed numerical ratios. No bias, $b=1.0$, is shown by the dashed line, whereas the solid line shows $b=0.6$. The lower panel shows the relations on logarithmic coordinates.

which shows that the exponent in ME is an estimate of $b \beta$ and not simply of $\beta$ alone. Thus, the exponent in ME confounds $b$ and $\beta$ and is only an estimate of the psychophysical exponent $\beta$ if $b=1$ - that is, if there is no response bias. Equation 9 shows that the effect of bias will be to attenuate or inflate the exponent, depending on whether $b$ is less than or greater than unity, respectively (the proportionality constant is affected as well; see below). Note that Equation 9 is consistent with Stevens's power law (i.e., Equation 1), and so the models cannot be distinguished in terms of fit; however, it will be shown below that there are patterns of results for the exponents that suggest that the model of bias given by Equation 8 is useful.

\section{Bias in Magnitude Production}

The model of bias is the same for MP as for ME, and so Equation 7 is generalized as

$$
\Psi / \Psi_{0}^{\prime}=\left(N / N_{0}\right)^{b^{\prime}},
$$

in which the prime on $b$ is used to indicate that the response bias is for a produced stimulus (i.e., $b^{\prime}$ indicates bias when a produced stimulus is the response variable, 
and $b$ indicates bias when produced numbers are the response variable). Equation 10 shows that the effect of bias in MP is exactly the same as in ME. If the bias parameter is less than unity, for example, numerical ratios greater or less than unity are judged as being less extreme (i.e., the participant does not produce sensation ratios as extreme as the number ratios), which again represents a response constriction. As shown by Equation 10, whether responses are constricted in one or both directions depends on where the reference number $N_{0}$ is located.

Substituting the psychophysical function (Equation 2) into Equation 10 and rearranging gives

$$
S^{\prime}=\left(S_{0}^{\prime} / N_{0}^{b^{\prime} / \beta}\right) N^{b^{\prime} / \beta},
$$

in which $S^{\prime}$ indicates that the stimulus intensity is produced by the participant (instead of being presented by the experimenter). Equation 11 shows that the exponent in MP (obtained from a regression of $\log S^{\prime}$ on $\log N$ ) is an estimate of $b^{\prime} / \beta$.

\section{Bias and the Regression Effect}

An immediate consequence of Equations 9 and 11 is that if the response bias tends to be less than unity, there will be a regression effect. Specifically, if the bias is less than unity, Equation 9 shows that the exponent for $\mathrm{ME}$ (i.e., $b \beta$ ) is attenuated, whereas Equation 11 shows that the exponent for MP obtained by taking the inverse (i.e., $\left.\beta / b^{\prime}\right)$ is inflated. Thus, Equations 9 and 11 provide a basis for Stevens's conjecture (e.g., 1986; Stevens \& Greenbaum, 1966) that the regression effect occurs because participants tend to constrict or shorten the range of the variable that they adjust, which is consistent with bias less than with unity.

Equations 9 and 11 also support Stevens's conjecture that the psychophysical exponent can be estimated by taking the geometric mean of the exponents for ME and MP. First, note that it is assumed in Stevens's conjecture that the response bias is equal across $\mathrm{ME}$ and $\mathrm{MP}$ - that is, $b^{\prime}=b$. It then follows from Equations 9 and 11 that taking the square root of the product of the ME exponent and inverse MP exponent gives ${ }^{2}$

$$
\sqrt{b \beta \times(\beta / b)}=\beta ;
$$

that is, the bias cancels out. Thus, the model of bias provides a formal basis for Stevens's conjecture to geometrically average the exponents from ME and MP. It also clarifies that an assumption of equal bias across the two procedures is made, as was in essence recognized by Stevens; "the question arises whether the error sources in magnitude estimation are exactly balanced by the error sources in magnitude production. Although exact balance may be possible, it seems hardly inevitable. One ought not to count too much on symmetry" (1986, pp. 271-272). Note that if a participant's bias differs across the two procedures, $b$ and $b^{\prime}$ do not cancel out and one does not obtain an estimate of $\beta$, but rather (from Equation 12) an estimate of $\beta \sqrt{ }\left(b / b^{\prime}\right)$; some examples of unequal bias are considered below.

Equations 9 and 11 also reveal an overlooked result. In particular, taking the square root of the ratio of the exponents provides an estimate of the magnitude of the bias,

$$
\sqrt{b \beta /(\beta / b)}=b
$$

Note that $b$ quantifies the magnitude of the regression effect, in that there is no effect if $b=1$ (in which case, the ME and inverse MP exponents are equal), whereas $b<1$ gives the usual regression effect. Again, if bias differs across the two conditions, only the geometric mean of the biases $\sqrt{ }\left(b \times b^{\prime}\right)$ can be estimated (which still provides useful information). With three or more conditions, however, one can estimate the separate biases, as shown below.

\section{Empirical Evidence: Magnitude Estimation and Magnitude Production}

Table 1 shows the results of applying Equations 12 and 13 to research where ME and MP were used in a counterbalanced design. In some cases, the exponents were reported and are shown in the table; in other cases, as indicated in the table notes, the exponents were estimated from graphs of the data; specifically, the exponents for the data from Stevens and Guirao (1962), Stevens and Greenbaum (1966), J. C. Stevens and Marks (1965), and Stevens (1986) were estimated from published graphs using Engauge Digitizer version 2.3 software, which can be freely downloaded at http://digitizer.sourceforge.net. In cases where the data were read from a graph, although the exponents are reported to two decimal places, little emphasis should be placed on the second decimal.

The top part of Table 1 shows that, for studies involving both ME and MP of loudness, the estimates of the bias $b$ obtained from Equation 13 are less than unity in all cases. In particular, the bias is small and is in the range of $0.8-0.9$. Note that larger bias (i.e., 0.8) indicates that the regression effect was larger, and in this way the bias parameter quantifies the magnitude of the regression effect. Bias less than unity implies that the psychophysical exponents were attenuated. Table 1 shows that corrected exponents $(\beta)$ obtained from Equation 12 are consistent across studies and are all in the range of 0.54-0.64, which is consistent with Stevens's (earlier) view that the psychophysical exponent for loudness is around 0.60 . Note that, although corrected psychophysical exponents for some of these studies have previously been discussed (e.g., by Stevens), the finding that the magnitude of the bias is also consistent across studies has not been noted (because Equation 13 has not been derived).

The next part of Table 1 shows results for studies involving ME and MP of duration. The table shows that the corrected psychophysical exponents for duration are close to unity, which suggests that the perception of du- 
Table 1

Parameter Estimates for Studies that Included Both Magnitude Estimation (ME) and Magnitude Production (MP) Conditions

\begin{tabular}{|c|c|c|c|c|c|}
\hline Study & $\begin{array}{c}\mathrm{ME} \\
b \beta\end{array}$ & $\begin{array}{l}\text { MP } \\
\beta / b\end{array}$ & $\beta$ & $b$ & Continuum \\
\hline \multicolumn{6}{|c|}{ Loudness } \\
\hline Stevens \& Guirao (1962) ${ }^{*}$ & 0.44 & 0.69 & 0.55 & 0.80 & noise \\
\hline Stevens \& Greenbaum $(1966)^{* *}$ & 0.54 & 0.66 & 0.60 & 0.90 & noise \\
\hline Green, Luce, \& Duncan (1977) & 0.44 & 0.65 & 0.54 & 0.82 & $1000-\mathrm{Hz}$ tone \\
\hline Hellman (1981) & 0.51 & 0.81 & 0.64 & 0.79 & $1000-\mathrm{Hz}$ tone \\
\hline & 0.59 & 0.70 & 0.64 & 0.92 & $3000-\mathrm{Hz}$ tone \\
\hline DeCarlo (2003) & 0.53 & 0.64 & 0.58 & 0.91 & $1000-\mathrm{Hz}$ tone \\
\hline \multicolumn{6}{|c|}{ Duration } \\
\hline Stevens \& Greenbaum (1966) & 0.87 & 1.20 & 1.02 & 0.85 & noise \\
\hline & 0.93 & 1.16 & 1.04 & 0.90 & light \\
\hline Painton, Cullinan, \& Mencke (1977) & 0.91 & 1.14 & 1.02 & 0.89 & $1000-\mathrm{Hz}$ tone \\
\hline
\end{tabular}

Notes $-b \beta$ is the exponent for ME, $\beta / b$ is the inverse of the exponent for MP. ${ }^{*}$ Data from Figure 11.6 of Gescheider, $1997 .{ }^{* *}$ Data from Figure 5.

ration is veridical. The table also shows that, although the psychophysical exponents $\beta$ for duration are considerably larger than those for loudness (i.e., 1.0 vs. 0.6 ), the bias is again generally small, in the range of $0.85-0.90$, which is consistent with the magnitude of the bias found for the loudness studies.

In summary, although corrected psychophysical exponents have been examined in prior research, response bias has not been examined, and so the results shown in Table 1 are new. The estimates of bias show a consistent pattern across well-known studies, in that the bias tends to be less than one, which is consistent with the view that participants tend to constrict or shorten the range of stimuli under their control. It should be noted that, although the magnitude of the bias shown in Table 1 is generally small, Stevens and Greenbaum (1966) also gave examples where the bias was large; some examples of large bias are presented below. Overall, the above derivations show that a power function generalization of the judgmental models of Equations 4 and 7 leads to models that are basically in line with several of Stevens's conjectures.

\section{Statistical Bias}

It is well known that, although one can obtain an unbiased estimate of the exponent for MP via a regression model, the inverse of the estimated exponent is biased. One can correct the estimate for statistical bias, however, as discussed by Thomas (1981). This was not done for the studies considered here because the information needed to provide the correction was not provided, except in the study of DeCarlo (2003). In that case, the statistical bias was estimated to be less than 0.01 , which is clearly too small to have any substantial effect on the conclusions; this is also the case for a cross-modality matching experiment reported by M. Teghtsoonian (1980; see below). Nevertheless, one can develop corrections for the estimates obtained by Equations 12 and 13. It would also be important to obtain standard errors for the estimates and perhaps perform a meta-analysis across studies. The purpose here, however, is to clarify basic mathematical consequences of the model of bias.

\section{Correlated Slopes and Intercepts}

Rule (1993) noted that any apparent relation between the slope $(\beta)$ and the intercept $(\log \alpha)$ across participants in magnitude scaling experiments is not psychologically meaningful, because the nature of the relation depends on the arbitrary units used for $S$; here I note that this follows directly from Equation 9. In particular, Equation 9 shows that if the estimated exponent varies across conditions or participants in ME (because of variation in the bias parameter $b$ across participants, for example), it follows that the intercept will have a negative relation to the exponent if $S_{0}>1$ (because $\alpha=N_{0} / S_{0}^{b \beta}$ gets smaller as $b \beta$ gets larger), will have a positive relation if $S_{0}<1$, and will have no relation if $S_{0}=1$, as was also noted by Rule. Note that this result does not follow from a power function generalization of Equation 3 (as used by others), which is basically a descriptive model of proportional judgment, but rather from a power function generalization of Equation 4, which is a process model of proportional judgment. With respect to MP, Equation 11 shows that the relation between the slope and intercept depends on the reference number used by the participant.

\section{Assimilation and the Regression Effect}

Cross (1973) proposed a perceptual mechanism for the regression effect. His proposal was based on the observation that, in ME of loudness, the previous stimulus intensity appears to have a small, positive effect on the current response, which is often referred to as "assimilation." Cross presented a regression model where assimilation had the effect of lowering the exponent, and in this way, assimilation could account for the regression effect. However, several studies have shown that the 
magnitude of assimilation appears to be too small to (fully) account for the regression effect (see DeCarlo, 2003), although, of course, it could contribute to it.

\section{Range Effects}

Several factors have been shown to affect the exponent obtained for Stevens's power law. Stevens and Greenbaum (1966), for example, noted that stimulus range affected the exponent; they also considered the possible contribution of stimulus range to the regression effect (see also Stevens, 1986). It is important to recognize that if judgment were strictly proportional, as in Equation 4, there would be no effect of stimulus range. Thus, the simple model of proportional judgment does not allow for variation in the exponent. In contrast, the bias model of Equation 8 can easily handle stimulus range effects, because it replaces the exponent $\beta$ of Stevens's power law in Equation 1 with $b \beta$, as shown by Equation 9. The implication is that what appear to be effects of stimulus range on the exponent of Equation 1 might actually be effects of stimulus range on the bias parameter $b$, and not the psychophysical exponent $\beta$. In my view, evidence for an effect of stimulus range on the bias parameter would clearly show the utility of the bias model with respect to uncovering overlooked patterns of results. Fortunately, data are available for experiments where both $\mathrm{ME}$ and MP were used in a counterbalanced design and where the stimulus range was varied.

Teghtsoonian and Teghtsoonian (1978; see also Teghtsoonian, 1973) conducted studies that showed that stimulus range affected the exponent of Stevens's power law. Loudness and distance were judged in ME and MP experiments where different ranges of stimuli were used (range was varied across groups of participants, with each participant participating in both ME and MP). Table 2 presents the results with respect to the exponents. The table shows that the ME exponent and the inverse MP exponent, which from Equations 9 and 11 are estimates of $b \beta$ and $\beta / b$, respectively, clearly vary with the stimulus range, as was also noted by Teghtsoonian and Teghtsoonian (1978). The estimates of $\beta$ derived from Equation 12 , on the other hand, are fairly constant as the range varies, for both the loudness and distance experiments. For example, the column for $\beta$ shows that the estimates of loudness are all around 0.67, which was Stevens's (later) estimate of the loudness exponent, whereas the distance exponents are all around 1.2 (Teghtsoonian \& Teghtsoonian, 1969, noted that the exponent for indoor distance appears to be greater than 1.0). Thus, Table 2 shows that, although the exponents for Stevens's power law vary systematically with the stimulus range, the corrected exponents do not.

The column for bias in Table 2 shows another important result, which is that the estimates of the bias vary systematically with the stimulus range. In particular, the bias increases (i.e., $b$ gets smaller) as the range increases; the effect is fairly large for loudness and is small at best for distance. Although Stevens (e.g., 1986) noted that participants generally appear to shorten the response
Table 2

Parameter Estimates for the Study of

Teghtsoonian and Teghtsoonian (1978)

\begin{tabular}{ccccc}
\multicolumn{5}{c}{ Teghtsoonian and Teghtsoonian (1978) } \\
\hline Log Range & ME & MP & & \\
\hline \multicolumn{5}{c}{ Loudness } \\
0.25 & 0.70 & 0.67 & 0.68 & 1.02 \\
0.50 & 0.52 & 0.90 & 0.68 & 0.76 \\
1.00 & 0.42 & 0.89 & 0.61 & 0.61 \\
2.00 & 0.41 & 1.18 & 0.70 & 0.59 \\
\multicolumn{5}{c}{ Distance } \\
0.25 & 1.31 & 1.06 & 1.18 & \\
0.50 & 1.23 & 1.14 & 1.18 & 1.11 \\
2.00 & 1.20 & 1.29 & 1.24 & 0.96 \\
\hline Note-Log range is the logarithm of the ratio of the largest to smallest
\end{tabular}

Note-Log range is the logarithm of the ratio of the largest to smallest stimulus. ME, magnitude estimation; MP, magnitude production.

range in magnitude scaling experiments, as shown in Table 1, Table 2 suggests that this occurs because experimenters have tended to use a wide stimulus range. In fact, for the smallest range in the distance study, the bias is expansive, $b>1$, which indicates a reversal of the regression effect, as was observed by Teghtsoonian and Teghtsoonian (1978). A useful aspect of the bias model is that it provides an interpretation of this reversal, attributing it to an effect of stimulus range on response bias, and not as an effect on the psychophysical exponent.

It can also be asked, as was done by a reviewer, why the stimulus range affects response bias. Several explanations have previously been offered in the literature. For example, some researchers have argued that participants tend to use a somewhat constant response range as the stimulus range varies (e.g., Jones \& Woskow, 1966; Poulton, 1979), which would account for the relation between stimulus range and bias, although this explanation has also been the subject of debate (e.g., see Poulton, 1979; Teghtsoonian, 1973). The purpose here is to present a formal model of bias, to show the consequences of bias, and to show how to measure and correct for the effects of bias; causal accounts of bias clearly require further investigation.

In summary, the model of bias presented here is consistent with stimulus range effects. In particular, the results shown in Table 2 suggest that the stimulus range affects the bias parameter $b$ but not the psychophysical exponent $\beta$. Thus, the bias model in this case appears to help disentangle effects of response factors from those of perception.

\section{Cross-Modality Matching}

This section shows that applying the model of bias to CMM leads to some important additional results. In CMM, participants adjust a stimulus on one continuum in response to a presented stimulus on another continuum. A model of proportional judgment in CMM is

$$
\Psi^{\prime} / \Psi_{0}^{\prime}=\Psi / \Psi_{0},
$$

which shows that participants produce sensation ratios that are proportional to those on the presented contin- 
uum (the prime again indicates the produced continuum). Note that CMM is conceptualized here as involving proportional judgment, as in Equations 4 and 7, and not as a matching task. In the present view, the participant in essence has to define what a "match" is by choosing a reference relation, $\alpha_{0}=\Psi_{0}^{\prime} / \Psi_{0}$, for the produced and presented sensations; the reference relation then serves as a reference point for comparative judgment, as in ME and MP, which are special cases of Equation 14.

As before, the above can be generalized through the use of a power function,

$$
\Psi^{\prime} / \Psi_{0}^{\prime}=\left(\Psi / \Psi_{0}\right)^{b^{\prime}},
$$

in which $b^{\prime}$ again indicates bias for a produced stimulus (as in MP above). Note that the response bias is modeled in the same way as it was for ME and MP above (i.e., Equations 8 and 10 are simply special cases of Equation 15). Substituting the psychophysical function (Equation 2) on each side of the above and rearranging gives

$$
S^{\prime}=\left(S_{0}^{\prime} / S_{0}^{b^{\prime} \beta / \beta^{\prime}}\right) S^{b^{\prime} \beta / \beta^{\prime}}
$$

in which $\beta$ is the exponent for the presented continuum and $\beta^{\prime}$ is the exponent for the produced continuum. Equation 16 clarifies how bias affects the exponent of Stevens's power law in CMM.

Note that one can perform a counterbalanced experiment, as for ME and MP, by switching which continuum is presented and which is produced in two CMM experiments. When the previously produced continuum is now the presented continuum, the exponent of Equation 16 is $b \beta^{\prime} / \beta$ (i.e., reverse the primes; compare with the derivation for MP above), in which $\beta^{\prime}$ is still the exponent for the originally produced (and now presented) continuum and $b$ is the response bias for that continuum. If it is assumed that the response bias is equal across the two CMM experiments - that is, $b=b^{\prime}-$ it follows that taking the geometric mean of the product of the first exponent and the inverse of the second exponent gives

$$
\sqrt{\left\{\left[\left(b^{\prime} \beta\right) / \beta^{\prime}\right] \times\left[\beta /\left(b^{\prime} \beta^{\prime}\right)\right]\right\}}=\beta / \beta^{\prime} .
$$

Equation 17 shows that, if one conducts counterbalanced CMM sessions, an estimate of the ratio of the exponents for each continuum is obtained. Although this was also
Stevens's conclusion, he derived this result using only the psychophysical function (i.e., Equation 2; see Stevens, $1966,1986)$, and so the role of response bias in CMM was not considered, although it was implicit in many of Stevens's arguments. The derivations presented here, on the other hand, explicitly consider bias and clarify assumptions and consequences of response bias in CMM.

As before, an overlooked result is that one can obtain an estimate of the bias by dividing the exponents from the two CMM experiments,

$$
\sqrt{\left\{\left[\left(b^{\prime} \beta\right) / \beta^{\prime}\right] /\left[\beta /\left(b^{\prime} \beta^{\prime}\right)\right]\right\}}=b^{\prime} .
$$

A number of researchers have run counterbalanced CMM sessions and have in essence used Equation 17 to get the ratio of psychophysical exponents (note that this means that they assumed equal bias across the two CMM sessions). But it has never been recognized, to my knowledge, that one can also obtain an estimate of the bias in CMM, as shown by Equation 18. Of course, one again has to consider possible effects of statistical bias in estimation, although the size of the bias again appears so small as to be inconsequential, as was also found for MP.

\section{Empirical Evidence: Cross-Modality Matching}

Table 3 presents several examples of CMM in which a counterbalanced design was used. The first is a wellknown study by J. C. Stevens and Marks (1965; see also Baird, 1997; Stevens \& Greenbaum, 1966). Participants adjusted the brightness of a white light to match the loudness of a band of noise and also matched loudness to brightness. The table shows results from Stevens and Marks's Figures 1 and 2. The third column of Table 3, which shows psychophysical exponents corrected for bias, shows that the estimate of the ratio of the brightnessto-loudness exponents from Equation 17 was around 1.0 in both experiments. Stevens (1986) suggested a value of the exponent for brightness as 0.66 and values for the exponent of loudness of 0.60 to 0.67 , and so the ratio of 1.0 is consistent with these values, as was also noted by Stevens and Marks; of course, it should be remembered that we have no information about the absolute magnitudes of the exponents in this case. Another interesting result is that the estimate of the bias is around 0.90 for

\begin{tabular}{|c|c|c|c|c|c|}
\hline Study & $b^{\prime} \beta / \beta^{\prime}$ & $\beta / b^{\prime} \beta^{\prime}$ & $\beta / \beta^{\prime}$ & $b^{\prime}$ & Judged/Produced \\
\hline J. C. Stevens \& Marks (1965)* & $\begin{array}{l}0.89 \\
0.96\end{array}$ & $\begin{array}{l}1.14 \\
1.08\end{array}$ & $\begin{array}{l}1.01 \\
1.02\end{array}$ & $\begin{array}{l}0.88 \\
0.94\end{array}$ & $\begin{array}{l}\text { brightness/loudness } \\
\text { brightness/loudness }\end{array}$ \\
\hline $\begin{array}{l}\text { Stevens \& Greenbaum }(1966)^{* *} \\
\text { Stevens }(1986)^{* * *}\end{array}$ & $\begin{array}{l}0.65 \\
0.53\end{array}$ & $\begin{array}{l}0.78 \\
0.63\end{array}$ & $\begin{array}{l}0.71 \\
0.58\end{array}$ & $\begin{array}{l}0.91 \\
0.92\end{array}$ & $\begin{array}{l}\text { loudness/vibration } \\
\text { loudness/vibration }\end{array}$ \\
\hline $\begin{array}{l}\text { Dawson \& Brinker (1971) } \\
\text { Wanschura \& Dawson (1974) } \\
\text { Lilienthal \& Dawson (1976) }\end{array}$ & $\begin{array}{l}0.44 \\
0.45 \\
0.40\end{array}$ & $\begin{array}{l}0.91 \\
0.81 \\
0.76\end{array}$ & $\begin{array}{l}0.63 \\
0.60 \\
0.55\end{array}$ & $\begin{array}{l}0.70 \\
0.75 \\
0.73\end{array}$ & $\begin{array}{l}\text { loudness/duration } \\
\text { loudness/duration } \\
\text { loudness/duration }\end{array}$ \\
\hline M. Teghtsoonian (1980) & 0.57 & 1.43 & 0.90 & 0.63 & loudness/length \\
\hline
\end{tabular}
both experiments, which is consistent with the bias found

Table 3

Parameter Estimates for Counterbalanced Cross-Modality Matching Experiments 
for ME and MP in Table 1. Thus, the results suggest that there was only small bias, and the bias was again less than unity.

The next rows of Table 3 show two CMM studies where a $60-\mathrm{Hz}$ vibration (applied to the middle finger) was matched to the loudness of a band of noise and vice versa (one study was reported in Stevens \& Greenbaum, 1966; the other is from Figure 33 of Stevens, 1986). In both cases, the bias is again small (around 0.9). With respect to the psychophysical exponent, Stevens (1986) claimed that the vibration exponent is close to 1.0 , and so the exponent ratios (i.e., loudness/vibration) of 0.71 and 0.58 are consistent with a loudness exponent in the range of 0.6-0.7, which is also consistent with the results shown in Table 1.

Next are three studies by Dawson and colleagues (Dawson \& Brinker, 1971; Lilienthal \& Dawson, 1976; Wanschura \& Dawson, 1974) in which loudness and duration were used in a counterbalanced design. The table shows that the exponent ratios, $\beta / \beta^{\prime}$, are again consistent with typical findings: the values are all around 0.6 , which is consistent with a loudness exponent of about 0.6 and a duration exponent of about 1.0. The bias, however, is somewhat larger (around 0.7) than that found in the other studies. The large bias indicates a large regression effect. The bias could have occurred because participants might have avoided using high levels of loudness or long durations when adjusting the stimuli (high levels or long durations might be aversive). Of course, this is just speculative, but if conditions with MP of duration and loudness were included, this could have been examined experimentally. Thus, the bias model helps to clarify which additional conditions would be the most informative.

The bottom of Table 3 shows results for an experiment by M. Teghtsoonian (1980). Adults and children of several age groups participated in two CMM sessions. In one session, length was matched to loudness, and in the other session loudness was matched to length. The results across children and adults were very similar, so only the average exponents are considered here (the results are the same when broken down by age group). For length produced in response to presented loudness, the exponent was 0.57 (i.e., the ratio of the sound exponent to the length exponent), whereas for loudness produced in response to presented length, the inverse of the exponent (of 0.70) was 1.43, as is shown in the table. Equation 17 in this case gives an estimate of $b^{\prime}$ of 0.63 , which reflects large bias and a large regression effect. Teghtsoonian (1980) also noted that the regression effect was sizable in this case; she also considered statistical estimation bias and found it to be no more than 0.01 (as was also found for MP by DeCarlo, 2003), so statistical bias in the estimates of the exponents obtained by Equations 17 and 18 does not appear to be an issue.

Equation 17 gives an estimate of $\beta / \beta^{\prime}$ for the data of Teghtsoonian of 0.9 , where $\beta$ is the exponent for loudness and $\beta^{\prime}$ is the exponent for length. We do not know the separate estimates of the exponents in this case, but a ratio of 0.9 is not consistent with prior findings. For example, for a loudness exponent of around 0.6 , as found in Table 1, and a length exponent around unity, as is often found (e.g., DeCarlo, 1994; Stevens, 1969, 1986), the predicted ratio would be around 0.6 , and so the obtained ratio of 0.9 is clearly larger than expected. Thus, in this case the simple model of (equal) bias does not account for the large value obtained for the exponent ratio $\beta / \beta^{\prime}$ in Teghtsoonian's study. This can be taken as evidence against the bias model or as evidence that an assumption should be relaxed.

With respect to the latter option, the results suggest that the bias might not have been equal across the two CMM conditions, and so the assumption $b=b^{\prime}$ should be relaxed. For example, when producing length in response to loudness, the exponent was 0.57 , which (for a length exponent close to 1.0) is consistent with the exponents of around 0.6 found for loudness in Table 1. On the other hand, the inverse exponent for producing loudness to length was 1.43 , as is shown in Table 3 , which is unexpectedly large. For example, the inverse exponent for producing loudness to numbers is typically around 0.7 , as shown in Table 1 , which is half as large as that found by Teghtsoonian. Thus, it appears that participants shortened their range of responses considerably more for produced loudness than for produced length, which in turn can help explain why a large value of 0.90 was obtained for the exponent ratio.

To illustrate, it can be shown that in the presence of unequal bias, Equation 17 gives an estimate of $\sqrt{ }\left(b^{\prime} / b\right)$ $\beta / \beta^{\prime}$ and Equation 18 gives an estimate of $\sqrt{ }\left(b^{\prime} \times b\right)$ (cf. the effects of unequal bias in ME and MP above). If the bias for length was small, say around 0.9 , and the bias for loudness was large, say 0.4 , the geometric mean would equal 0.6 , which is consistent with the estimate of bias of 0.63 shown in Table 3 . The exponent ratio would then be inflated by a factor of $\sqrt{ }\left(b^{\prime} / b\right)=\sqrt{ }(0.9 / 0.4)=1.5$. Thus, if the loudness/length exponent ratio was around 0.6 (i.e., a loudness exponent of 0.6 and a length exponent of $1.0)$, the inflated exponent ratio would be $0.6 \times 1.5=$ 0.9 , which is the same as the exponent shown in Table 3 . Thus, relaxing the assumption of equal bias across the two CMM conditions can account for Teghtsoonian's results. $^{3}$ To help validate this account, however, it would have been important to have at least one additional condition, such as MP of loudness to numbers - if a largerthan-expected inverse exponent were found, as Teghtsoonian found for CMM of loudness to length, that would suggest that there was indeed large bias for loudness production (and one could look for sources of the bias in details of the experiment); if not, either the bias model is wrong or it needs to be revised (another possibility is that the bias is not stable across sessions). Here, I simply note that the model of bias helps to clarify the findings and also indicates the kind of experiments that are needed to help answer relevant questions. 


\section{On Predicting Exponents in Magnitude Scaling}

If, in fact, there are stable psychophysical exponents (i.e., $\beta$ ) for different continua, as argued by Stevens, then one can make predictions about the exponents across different experiments. For example, in an article in which data of Mashhour and Hosman (1968) were analyzed, Stevens (1969), using the exponents obtained from 7 ME experiments, was able to predict, to an extent, the exponents obtained in $17 \mathrm{CMM}$ experiments. Stevens viewed this as important validation evidence for magnitude scaling (see also Stevens, 1960, 1966, 1986). This section shows that the models of bias in ME, MP, and CMM presented here are important because they clarify exactly when bias will or will not be removed when predicting exponents across experiments and with what assumptions. For example, it is shown that if one has two ME experiments, one can directly measure the response bias in a CMM experiment, with no assumptions about the CMM bias. This new result is examined using data discussed by Stevens (1969).

Consider taking the ratio of two ME exponents in order to predict a CMM exponent. From Equation 9, the ME exponents are each estimates of $b \beta$-that is, the psychophysical exponent $\beta$ for each continuum contaminated by response bias $b$. Note that if it is assumed that the response bias is the same across two ME experiments where different continua are judged, then the bias $b$ cancels out of the exponent ratio. ${ }^{4}$ Thus, with the assumption of equal response bias, the bias parameter is removed when two ME experiments are used to predict a CMM exponent. This, in turn, means that the exponents given in Stevens's (1969) Table 2, which were predicted from ME experiments, do not need to be corrected for the regression effect, contrary to Stevens's claim. ${ }^{5}$ This shows why it is important to specify the model of bias explicitly and to derive its implications.

Another important aspect of the above derivations is that they suggest that there is additional information in Stevens's (1969) Table 2. In particular, in addition to exponents predicted from ME experiments, Stevens presented exponents from (confirmatory) CMM experiments. An interesting consequence of the above results is that they show that one can estimate the bias for the produced response in CMM. In particular, it follows from Equation 9, with the assumption of equal number bias $b$ across the two ME sessions, that the ratio of ME exponents provides an estimate of $\beta / \beta^{\prime}$, as noted above. As shown by Equation 16, the exponent obtained in a CMM experiment provides an estimate of $b^{\prime} \beta / \beta^{\prime}$ - that is, the CMM exponent (predicted from ME to be $\beta / \beta^{\prime}$ ) contaminated by response bias $b^{\prime}$. It follows that dividing the obtained CMM exponent $\left(b^{\prime} \beta / \beta^{\prime}\right)$ by the ratio predicted from ME $\left(\beta / \beta^{\prime}\right)$ gives an estimate of the bias $b^{\prime}$ for the produced response in CMM. Note that this only involves an assumption of equal bias across the two ME experiments; it does not involve any assumptions about the bias $b^{\prime}$ in CMM. This result suggests a new way to look at the exponents presented in Stevens's (1969) Table 2.
Table 4 shows estimates of bias obtained in this manner for the 17 CMM experiments of Mashhour and Hosman (1968; as given in Table 2 of Stevens, 1969), organized by the produced continuum, with the average bias for each produced continuum also shown. An interesting pattern of results is immediately apparent, in that the bias tends to be consistent within each response continuum as the judged continuum varies. For example, the top part of Table 4 shows that the response bias when length was the produced continuum is generally small, with an average of 0.96 , as loudness of noise, duration, weight, lightness of grays, and surface area were judged. Thus, the response bias for produced length appears to be small (i.e., is close to unity). The next part of Table 4 shows that the bias is larger when duration was the response variable (average of 0.80 ). Next, a pattern is clear for finger span, in that the bias is quite large across all five CMM experiments, with an average of 0.62 . The bias when noise was the response variable is also consistent across experiments but differs from that found for the other continua in that it is consistently greater than unity, with an average of 1.24; Stevens (1969) noted that the results for ME of noise in Mashhour and Hosman's experiment were unusual, in terms of the psychophysical exponent, most likely because a narrow range of noise was used, whereas Table 4 suggests that, when noise was the produced continuum, the results were also unusual with respect to response bias (again most likely due to peculiarities of the procedure).

In summary, Table 4 shows a clear pattern of results with respect to response bias in the experiments of Mashhour and Hosman (1968) that has been overlooked up to this point. In particular, there appears to be consistent bias for each response continuum as the judged continuum varies. For example, when finger span was the response variable, the bias was consistently large across all

Table 4

Bias Parameter Estimates for 17 CMM Experiments Discussed by Stevens (1969)

\begin{tabular}{|c|c|c|c|}
\hline \multicolumn{2}{|c|}{ Continuum } & \multirow[b]{2}{*}{$b^{\prime}$} & \multirow[b]{2}{*}{ Average $b^{\prime}$} \\
\hline Produced & Judged & & \\
\hline length & noise & 0.92 & 0.96 \\
\hline length & duration & 1.09 & \\
\hline length & weight & 0.85 & \\
\hline length & gray & 1.14 & \\
\hline length & surface & 0.80 & \\
\hline duration & noise & 0.88 & 0.80 \\
\hline duration & weight & 0.73 & \\
\hline duration & gray & 0.88 & \\
\hline duration & surface & 0.71 & \\
\hline finger span & noise & 0.69 & 0.62 \\
\hline finger span & duration & 0.63 & \\
\hline finger span & length & 0.60 & \\
\hline finger span & gray & 0.72 & \\
\hline finger span & surface & 0.48 & \\
\hline noise & weight & 1.17 & 1.24 \\
\hline noise & gray & 1.47 & \\
\hline noise & surface & 1.09 & \\
\hline
\end{tabular}


of the judged continua. Thus, Table 4 provides evidence that the deviations from predicted exponents in CMM are not simply random but rather show a systematic pattern across different response modalities. Again, without explicit derivations of the effects of bias in ME, MP, and CMM, one would not know to look for this result.

\section{SUMMARY AND CONCLUSIONS}

The simple model of bias presented here shows a specific way in which to relax the assumption of proportional judgment in magnitude scaling. In particular, a power function generalization of a process version of the judgmental model is shown to encompass various aspects of response bias, which generally can be viewed as peculiarities of response usage in ME, MP, and CMM. The approach is shown to fill some gaps in the theory of magnitude scaling. For example, it provides a formal basis for Stevens's conjecture that an estimate of the psychophysical exponent can be obtained from the geometric mean of ME and (inverse) MP exponents. In addition, a new result is that an estimate of the bias can be obtained from the square root of the ratio of exponents. It is shown that obtaining an estimate of the bias in this way is informative. The model of bias also clarifies the meaning of the proportionality constant and the relation between the exponent and intercept; the model is also shown to be consistent with range effects. Consequences of unequal biases are also discussed, and it is shown that, with appropriate experiments, one can obtain information about differences in bias across different response continua. For example, analysis of data presented by Stevens (1969) provides evidence of consistent response bias across different judged continua. The approach presented here provides a starting point for additional research and also clarifies the type of validation studies that need to be done.

Of course, judgments can also deviate from proportionality in more complex ways than considered here. For example, a general judgmental model for ME can be written as

$$
g\left(N, N_{0}\right)=f\left(\Psi, \Psi_{0}\right) .
$$

in which $g$ and $f$ are functions. The above simply states that response relations reflect sensation relations; Equation 4 is a special case in which $g$ and $f$ are ratios, whereas Equation 8 is a special case in which $f$ is a power function of ratios. The above can be further generalized by recognizing that participants can use more than just $N_{0}$ and $\Psi_{0}$ as reference points (e.g., see DeCarlo, 1990, 1994, 2003). Nevertheless, the simple power generalization considered here appears to be useful and has a number of implications for psychophysical theory that up to this point have not been fully appreciated.

\section{REFERENCES}

BAIRD, J. C. (1997). Sensation and judgment: Complementarity theory of psychophysics. Mahwah, NJ: Erlbaum.
Cross, D. V. (1973). Sequential dependencies and regression in psychophysical judgments. Perception \& Psychophysics, 14, 547-552.

Cross, D. V. (1974). Some technical notes on psychophysical scaling. In H. R. Moskowitz, B. Scharf, \& J. C. Stevens (Eds.), Sensation and measurement: Papers in honor of S. S. Stevens (pp. 23-36). Dordrecht: D. Reidel.

Curtis, D. W., Attneave, F., \& Harrington, T. L. (1968). A test of a two-stage model of magnitude judgment. Perception \& Psychophysics, 3, 25-31.

DaWson, W. E., \& BRINKER, R. P. (1971). Validation of ratio scales of opinion by multimodality matching. Perception \& Psychophysics, $\mathbf{9}$, 413-417.

DeCarlo, L. T. (1990). Sequential effects in magnitude scaling (Doctoral dissertation, SUNY at Stony Brook, 1989). Dissertation Abstracts International, 50, 3730B.

DeCARLo, L. T. (1994). A dynamic theory of proportional judgment: Context and judgment of length, heaviness, and roughness. Journal of Experimental Psychology: Human Perception \& Performance, 20, 372-381.

DeCarlo, L. T. (2003). An application of a dynamic model of judgment to magnitude production. Perception \& Psychophysics, 65, 152162.

DeCarlo, L. T., \& Cross, D. V. (1990). Sequential effects in magnitude scaling: Models and theory. Journal of Experimental Psychology: General, 119, 375-396.

Gescheider, G. A. (1997). Psychophysics: The fundamentals (3rd ed.). Hillsdale, NJ: Erlbaum.

Green, D. M., Luce, R. D., \& Duncan, J. E. (1977). Variability and sequential effects in magnitude production and estimation of auditory intensity. Perception \& Psychophysics, 22, 450-456.

Hellman, R. P. (1981). Stability of individual loudness functions obtained by magnitude estimation and production. Perception \& Psychophysics, 29, 63-70.

JoNES, F. N., \& Woskow, M. J. (1966). Some effects of context on the slope in magnitude estimation. Journal of Experimental Psychology, 71, 177-180.

LiLienthal, M. G., \& Dawson, W. E. (1976). Inverse cross-modality matching: A test of ratio judgment consistency for group and individual data. Perception \& Psychophysics, 19, 252-260.

Mashiour, M., \& Hosman, J. (1968). On the new "psychophysical law": A validation study. Perception \& Psychophysics, 3, 367-375.

Painton, S. W., Cullinan, W. L., \& MencKe, E. O. (1977). Individual pitch functions and pitch-duration cross-dimensional matching. Perception \& Psychophysics, 21, 469-476.

Poulton, E. C. (1979). Models for biases in judging sensory magnitudes. Psychological Bulletin, 86, 777-803.

Rule, S. J. (1993). Analyzing coefficients of psychophysical power functions. Perception \& Psychophysics, 54, 439-445.

Stevens, J. C., \& Marks, L. E. (1965). Cross-modality matching of brightness and loudness. Proceedings of the National Academy of Sciences, 54, 407-411.

Stevens, S. S. (1960). The psychophysics of sensory function. American Scientist, 48, 226-253.

Stevens, S. S. (1966). Matching functions between loudness and ten other continua. Perception \& Psychophysics, 1, 5-8.

Stevens, S. S. (1969). On predicting exponents for cross-modality matches. Perception \& Psychophysics, 6, 251-256.

Stevens, S. S. (1986). Psychophysics. New Brunswick, NJ: Transaction Books.

Stevens, S. S., \& Greenbaum, H. B. (1966). Regression effect in psychophysical judgment. Perception \& Psychophysics, 1, 439-446.

Stevens, S. S., \& Guirao, M. (1962). Loudness, reciprocality, and partition scales. Journal of the Acoustical Society of America, 34, 14661471.

Teghtsoonian, M. (1980). Children's scales of length and loudness: A developmental application of cross-modal matching. Journal of Experimental Child Psychology, 30, 290-307.

Teghtsoonian, M., \& Teghtsoonian, R. (1969). Scaling apparent distance in natural indoor settings. Psychonomic Science, 16, 281-283.

Teghtsoonian, R. (1973). Range effects in psychophysical scaling and a revision of Stevens' law. American Journal of Psychology, 86, 3-27. 
Teghtsoonian, R., \& Teghtsoonian, M. (1978). Range and regression effects in magnitude scaling. Perception \& Psychophysics, 24, 305-314.

Thomas, H. (1981). Estimation in the power law. Psychometrika, 46, 29-34. Wanschura, R. G., \& Dawson, W. E. (1974). Regression effect and individual power functions over sessions. Journal of Experimental Psychology, 102, 806-812.

\section{NOTES}

1. Note that no assumptions are made as to whether or not the participant is consciously aware of the reference points that are used.

2. Of course it is simpler to just divide the ME exponent by the MP exponent, but here I am showing the connection to Stevens's approach.

3. Clearly, there are more unknowns (the parameters $\beta / \beta^{\prime}, b$, and $b^{\prime}$ ) than knowns (the two estimates of the exponent from the two CMM ex- periments) and so one cannot obtain unique estimates of the parameters (i.e., they are not identified). As is shown in the last section, with three conditions one can solve for unequal biases $\left(b\right.$ and $\left.b^{\prime}\right)$ and the ratio of exponents $\left(\beta / \beta^{\prime}\right)$.

4. In my view, it seems quite reasonable to assume that bias in number usage might be the same across two ME experiments with the same participants. If the biases are not equal, Equation 9 shows that the CMM exponent is multiplied by the ratio of biases.

5. In this case, Stevens used exponents from MP to correct for the regression effect. Suffice it to say that it should be clear from the above that this does not remove the bias.

(Manuscript received April 26, 2004; revision accepted for publication November 1, 2004.) 